\title{
CALCULATION OF ALL EIGENVALUES OF A REAL MATRIX
}

1. Procedure declaration. The procedure $Q R$ finds all (also complex) eigenvalues of real matrices.

Data: $\begin{aligned} n & - \text { dimension of matrix } a \\ a[1: n, 1: n] & - \text { matrix whose eigenvalues are to be calculated, } \\ p & - \text { number of significant bits representing the mantissa } \\ & \text { in actual implementation of Algol. }\end{aligned}$

Results:

On exit from procedure $Q R$ the real parts of the eigenvalues are placed in the first column of $a(a[i, 1]$ for $i=1,2, \ldots, n)$ and the imaginary parts of the eigenvalues are placed in the second column of that array $(a[i, 2]$ for $i=1,2, \ldots, n)$. All initial elements of $a$ are destroyed.

2. Method used. This procedure is written on the basis of Francis' iterative $Q R$ method (1961) [1]:

$$
\begin{aligned}
& A=A_{1}=Q_{1} R_{1} \quad R_{1} Q_{1}=A_{2} \\
& A_{2}=Q_{2} R_{2} \quad R_{2} Q_{2}=A_{3}
\end{aligned}
$$

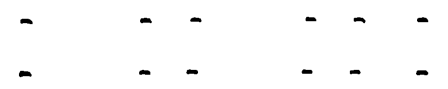

$$
\begin{aligned}
& A_{\mathrm{s}}=Q_{\mathrm{s}} R_{\mathrm{s}} \quad R_{\mathrm{s}} Q_{\mathrm{s}}=A_{\mathrm{s}+1},
\end{aligned}
$$

where $Q_{k}$ are orthogonal matrices, and $R_{k}$ are upper triangle matrices.

After entry to procedure $Q R$ the matrix $A$ is converted to the form of the upper triangular matrix of Hessenberg and practically the next iterations are processed on matrices of that form.

The transformation of matrix $A$ to the form of the upper triangular matrix of Hessenberg and further iterations in this procedure are based on Householder's method. 


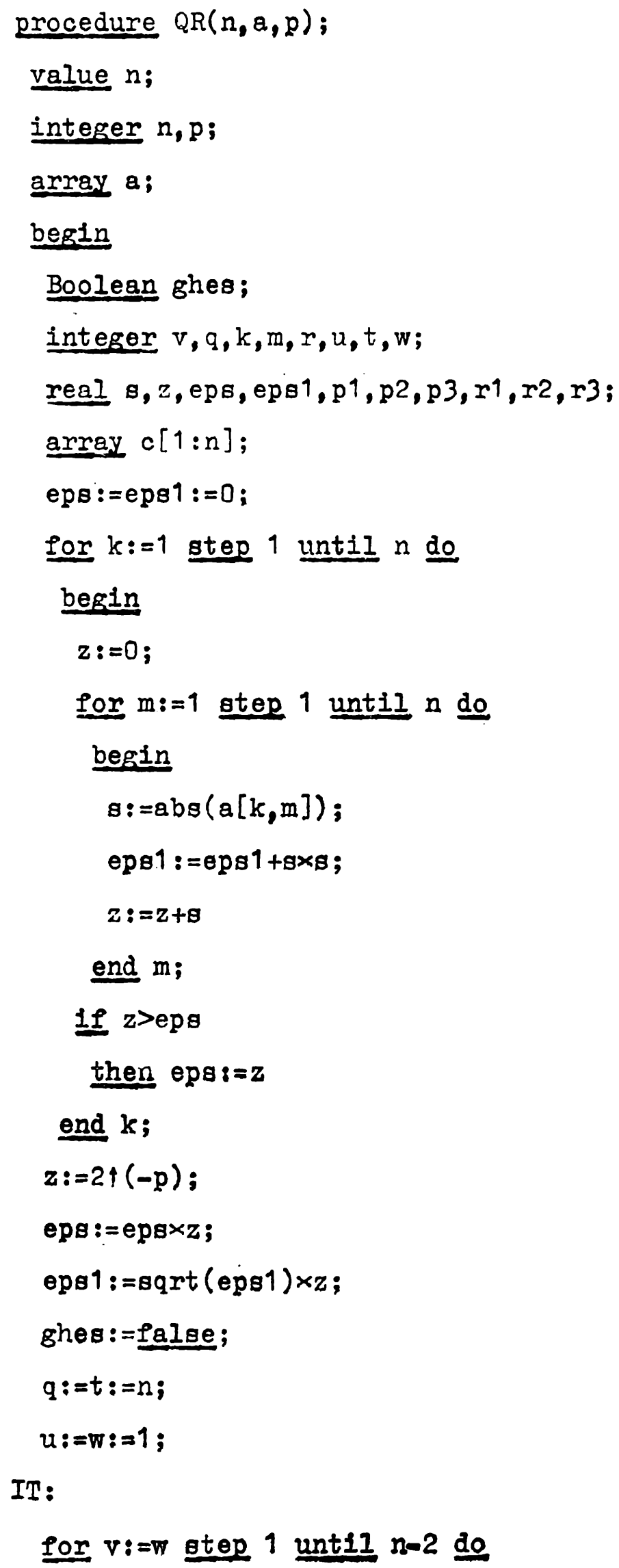


begin

\section{$r:=v+1 ;$ \\ if ghes \\ then \\ begin}

$\mathrm{q}:=$ if $\mathrm{v}=\mathrm{n}-2$ then $\mathrm{n}$ else $\mathrm{v}+3$;

if $\nabla<n-3$

then

begin

$t:=\mathrm{v}+4$;

$a[t, r]:=a[t, r+1]:=.0$

end $v<n-3$

else $t:=n$

end ghes;

if $\neg(v=w \wedge$ ghes $)$

then

begin

$z:=.0 ;$

for $k:=q$ step -1 until $r$ do

begin

$$
\begin{aligned}
& r 1:=c[k]:=a[k, v] ; \\
& z:=z+r 1 \times r 1
\end{aligned}
$$

end $k$

$$
\text { end } \neg(v=w \wedge \text { ghes }) \text {; }
$$

$s:=\operatorname{sqrt}(z)$;

if $\neg$ (s>epsVghes)

then go to $d v$;

if $x 1<0$

then $s:=-s$ : 


$$
r 1:=1.0 /(r 1+s) ;
$$

$z:=1.0 ;$

for $k:=r+1$ step 1 unt 11 $q$ do

begin

$$
\begin{aligned}
& \mathrm{r} 2:=c[\mathrm{k}]:=c[\mathrm{k}] \times \mathrm{r}^{1} ; \\
& \mathrm{z}:=\mathrm{z}+\mathrm{r} 2 \times \mathrm{r} 2
\end{aligned}
$$

end $k$;

$z:=2.0 / z$;

for $m:=r$ step 1 until $n$ do

begin

$$
\begin{aligned}
& r 1:=a[r, n] ; \\
& \text { for } k:=r+1 \text { gtep } 1 \text { unti] } q \text { do } \\
& r 1:=r 1+c[k] \times a[k, m] ; \\
& r 1:=r 1 \times z ; \\
& a[r, m]:=a[r, m]-r 1 ; \\
& \text { for } k:=r+1 \text { step } 1 \text { unti1 q do } \\
& a[k, m]:=a[k, m]-c[k] \times r 1
\end{aligned}
$$

end m;

for $m:=u$ step 1 unt11 $t$ do

\section{begin}

$$
\begin{aligned}
& r 1:=a[\mathrm{~m}, r] ; \\
& \text { for } k:=r+1 \text { step } 1 \text { unti] } q \text { do } \\
& r 1:=r 1+a[\mathrm{~m}, \mathrm{k}] \times c[k] ; \\
& r 1:=r 1 \times z ; \\
& a[m, r]:=a[\mathrm{~m}, r]-r 1 ; \\
& \text { for } k:=r+1 \text { step } 1 \text { until q do } \\
& a[m, k]:=a[m, k]-c[k] \times r 1
\end{aligned}
$$

end $\mathrm{m}$;

if $\neg(v=w \wedge$ ghe $\theta)$

then $a[r, v]:=-8$; 


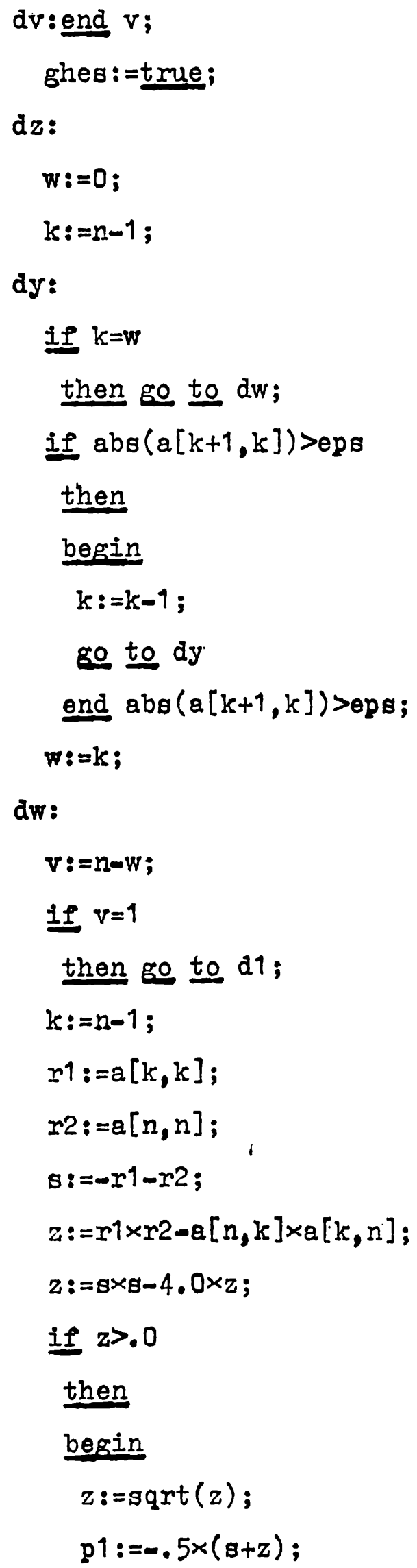




$$
\begin{aligned}
& p 2:=-.5 \times(8-z) ; \\
& p 3:=.0 \\
& \text { end } z>.0 \\
& \text { else }
\end{aligned}
$$

begin

$$
\begin{aligned}
& z:=\operatorname{gqrt}(-z) ; \\
& \mathrm{p} 1:=\mathrm{p} 2:=-.5 \times 8 ; \\
& \mathrm{p} 3:=.5 \times \mathrm{z}
\end{aligned}
$$

end $z \leq .0$;

if $v=2$

then go to d2;

$\mathrm{m}:=\mathrm{n}-2 ;$

red2:

$$
\begin{aligned}
& u:=m ; \\
& m:=m-1 ; \\
& k:=m+2 ; \\
& r:=m+3 ; \\
& r 1:=a[u, u] ; \\
& z:=r 1-p 1-p 2 ; \\
& s:=a[k, u] ; \\
& r 3:=p 1 \times p 2+p 3 \times p 3 ; \\
& r 1:=r 1 \times z+a[u, k] \times 8+r 3 ; \\
& r 2:=c[k]:=8 \times(z+a[k, k]) ; \\
& r 3:=c[r]:=a[r, k] \times s ; \\
& i f m=w
\end{aligned}
$$

then

defl:

\section{begin}

$$
\begin{aligned}
& z:=r 1 \times r 1+r 2 \times r 2+r 3 \times r 3 ; \\
& a[r, u]:=.0 ;
\end{aligned}
$$


$\mathrm{w}:=\mathrm{m}$;

go to IT

end $m=w$;

if $a b s(x 1)$ <eps

then go to red2

else

if $a b_{B}\left(a[u, m] \times\left(a b_{B}(r 2)+a b_{B}(r 3)\right) / r 1\right) \leq e p s 1$

then go to defl

else go to red2;

d2;

$a[n, 1]:=p 1 ;$

$a[n, 2]:=p 3$;

$a[k, 1]:=p 2$;

$a[k, 2]:=-p 3 ;$

$n:=n-2 ;$

go to d 3 ;

d1:

$a[n, 1]:=a[n, n] ;$

$a[n, 2]:=.0$;

$n:=n-1$;

d3:

if $n \geq 1$

then go to $\mathrm{dz}$

end $Q R$

3. Certification. The algorithm $Q R$ has been verified on the Odra 1204 computer. Results of the control calculations are given in the table.

With the matrix given

$\begin{array}{llllr}0 & 0 & 0 & 0 & -1 \\ 1 & 0 & 0 & 0 & 0 \\ 0 & 1 & 0 & 0 & -1 \\ 0 & 0 & 1 & 0 & -1 \\ 0 & 0 & 0 & 1 & 0\end{array}$


one obtains the following results:

TABLE

The eigenvalues exact Calculated eigenvalues

$$
\begin{array}{ll}
-1 & -0.9999999998 \\
0.5+0.86602540378 \times i & 0.5+0.8660254039 \times i \\
0.5-0.86602540378 \times i & 0.5-0.8660254039 \times i \\
-i & 0.0-1.0000000000 \times i \\
i & 0.0+1.0000000000 \times i
\end{array}
$$

\section{References}

[1] J. G. F. Francis, The $Q R$ transformation, Parts 1 and 2, Computer J. 4 (1961), p. 256 and p. 332 .

CENTRALNE LABORATORIUM GAZOWNICTWA, WARSZAWA

Received on 24. 6. 1970;

revised version on 29. 1. 1972

ALGORYTM 19

A. RAKUS (Waramawa)

\section{OBLICZANIE WSZYSTKICH WARTOSCI WLASNYCH MACIERZY} RZECZY WISTEJ

\section{ST RESZCZENIE}

Procedura $Q R$ wyznacza wszystkie wartości własne macierzy rzeczywistej. Dane:

$n$ - wymiar macierzy $a$,

$a[1: n, 1: n]$ - macierz, której wartości własne mają byc obliczone,

$p$ - ilość bitów przeznaczonych na mantysę liczby zmiennoprzecinkowej w maszynie, na której przeprowadzamy obliczenia.

Wyniki :

Po zakończeniu obliczeń, wartości własne są umieszczone na miejscach $a[i, 1]$ oraz $a[i, 2](i=1,2, \ldots, n)$, gdzie $a[i, 1]$ jest częścią rzeczywistą, natomiast $a[i, 2]$ częśsią urojoną $i$-tej wartości własnej.

Cała macierz $a$ jest zniszczona.

Procedura napisana jest na podstawie metody $Q R$ (Francis [1]). Algorytm podany $w$ tej pracy sprowadza macierz do postaci górnej Hessenberga i dalsze iteracje przebiegają na macierzy tej postaci.

Wstępne sprowadzenie do postaci górnej Hessenberga, jak i postępowanie iteracyjne, oparte są na metodzie Householdera. Obliczenia kontrolne, wykonane na maszynie cyfrowej Odra 1204, wykazały poprawnośc procedury. 